\title{
REFERENCES
}

500

501

502

503

504

505

506

507

508

509

510

512

513

514

515

516

517

518

519

520

521

Bear, J., 1972. Dynamics of fluids in porous media Elsevier, New York, New York.

Birkholzer, J., G.M. Li, C.F. Tsang, and Y. Tsang, 1999. Modeling studies and analysis of seepage into drifts at yucca mountain. Journal of Contaminant Hydrology 38:349-384.

Bodvarsson, G.S., W. Boyle, R. Patterson, and D. Williams, 1999. Overview of scientific investigations at yucca mountain - the potential repository for high-level nuclear waste. Journal of Contaminant Hydrology 38:3-24.

Deutsch, C.V., and A.G. Journel, 1992. Gslib: Geostatistical software library and user's guide Oxford University Press, New York, New York.

Finsterle, S. 1999. Itough2 user 's guide Report No. LBNL-40040. Lawrence Berkeley National Laboratory.

Finsterle, S., 2000. Using the continuum approach to model unsaturated flow in fractured rock. Water Resources Research 36:2055-2066.

Finsterle, S., and R.C. Trautz, 2001. Numerical modeling of seepage into underground openings. Mining Engineering 53:52-56.

Finsterle, S., C.F. Ahlers, R.C. Trautz, and P.J. Cook, 2003. Inverse and predictive modeling of seepage into underground openings. Journal of Contaminant Hydrology 62-63:89-109.

Fujimaki, H., and M. Inoue, 2003. A transient evaporation method for determining soil hydraulic properties at low pressure. Vadose Zone J 2:400-408.

Ho, C.K., 1997. Evaporation of pendant water droplets in fractures. Water Resources Research 33:2665-2671.

Knight, J.H., J.R. Philip, and R.T. Waechter, 1989. The seepage exclusion problem for spherical cavities. Water Resources Research 25:29-37. 
522

523

524

525

526

527

528

529

530

531

532

533

534

535

LeCain, G.D. 1995. Pneumatic testing in 45-degree-inclined boreholes in ash-flow tuff near superior, arizona Water-Resources Investigations Report 95-4073. U.S. Geological Survey, Denver, Colorado.

Li, G.M., and C.-F. Tsang, 2003. Seepage into drifts with mechanical degradation. Journal of Contaminant Hydrology 62:157-172.

Murray, F.W., 1966. On the computation of saturation vapor pressure. J. Appl. Meteor. 6:204.

Or, D., and T.A. Ghezzehei, 2000. Dripping into subterranean cavities from unsaturated fractures under evaporative conditions. Water Resources Research 36:381-393.

Penman, H.L., 1948. Natural evaporation from open water, bare soil and grass. Proceedings of the Royal Society of London. Series A, Mathematical and Physical Sciences 193:120-145.

Philip, J.R., 1989a. Asymptotic solutions of the seepage exclusion problem for ellipticcylindrical, spheroidal, and strip-shaped and disc- shaped cavities. Water Resources Research 25:1531-1540.

Philip, J.R., 1989b. The seepage exclusion problem for sloping cylindrical cavities. Water Resources Research 25:1447-1448.

Philip, J.R., J.H. Knight, and R.T. Waechter, 1989a. The seepage exclusion problem for parabolic and paraboloidal cavities. Water Resources Research 25:605-618.

Philip, J.R., J.H. Knight, and R.T. Waechter, 1989b. Unsaturated seepage and subterranean holes - conspectus, and exclusion problem for circular cylindrical cavities. Water Resources Research 25:16-28.

Pruess, K., C. Oldenburg, and G. Moridis. 1999. Tough2 user's guide, version 2.0 LBNL-43134. Lawrence Berkeley National Laboratory, Berkeley, Calif.

Rohsenow, W.M., and H. Choi, 1961. Heat, mass and momentum transfer Prentice-Hall Inc., Englewood Cliffs, New Jersey. 
546 Trautz, R.C., and J.S.Y. Wang, 2001. Evaluation of seepage into an underground opening using 547 small- scale field experiments, yucca mountain, nevada. Mining Engineering 53:41-44.

548 Trautz, R.C., and J.S.Y. Wang, 2002. Seepage into an underground opening constructed in unsaturated fractured rock under evaporative conditions. Water Resources Research $550 \quad 38: 1188$.

551 van Genuchten, M.T., 1980. A closed-form equation for predicting the hydraulic conductivity of 552 unsaturated soils. Soil Science Society of America Journal 44:892-898.

553 Vargaftik, N.B., 1975. Tables on the thermophysical properties of liquids and gases. 2nd ed. $554 \quad$ John Wiley \& Sons, New York, NY.

555 Wang, J.S.Y., R.C. Trautz, P.J. Cook, S. Finsterle, A.L. James, and J. Birkholzer, 1999. Field 556 tests and model analyses of seepage into drift. Journal of Contaminant Hydrology 38:323$557 \quad 347$.

558 Zhang, J.T., and B.X. Wang, 2002. Effect of capillarity at liquid-vapor interface on phase change 559 without surfactant. International Journal of Heat \& Mass Transfer 45:2689-2694.

560 Zhang, J.T., B.X. Wang, and X.F. Peng, 2001. Thermodynamic aspect of the shift of concave 561 liquid-vapor interfacial phase equilibrium temperature and its effect on bubble formation. 562 International Journal of Heat and Mass Transfer 44:1681-1686. 


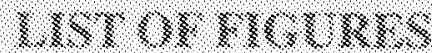

(4) A Ar velosity

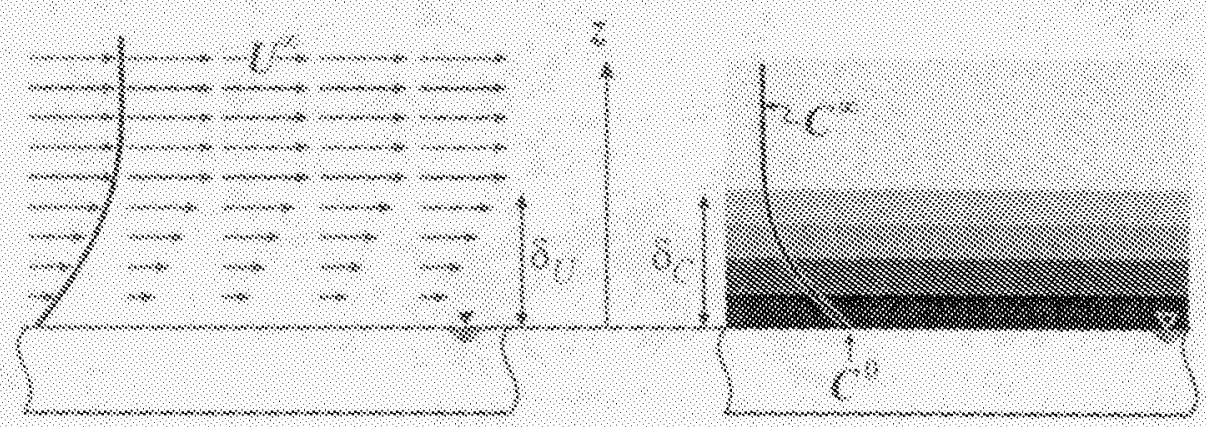

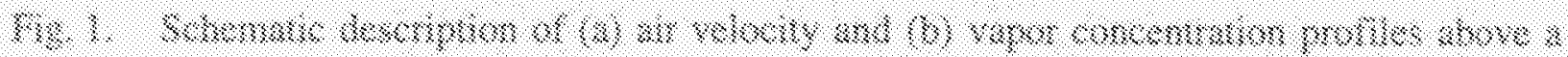

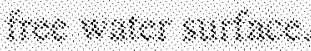



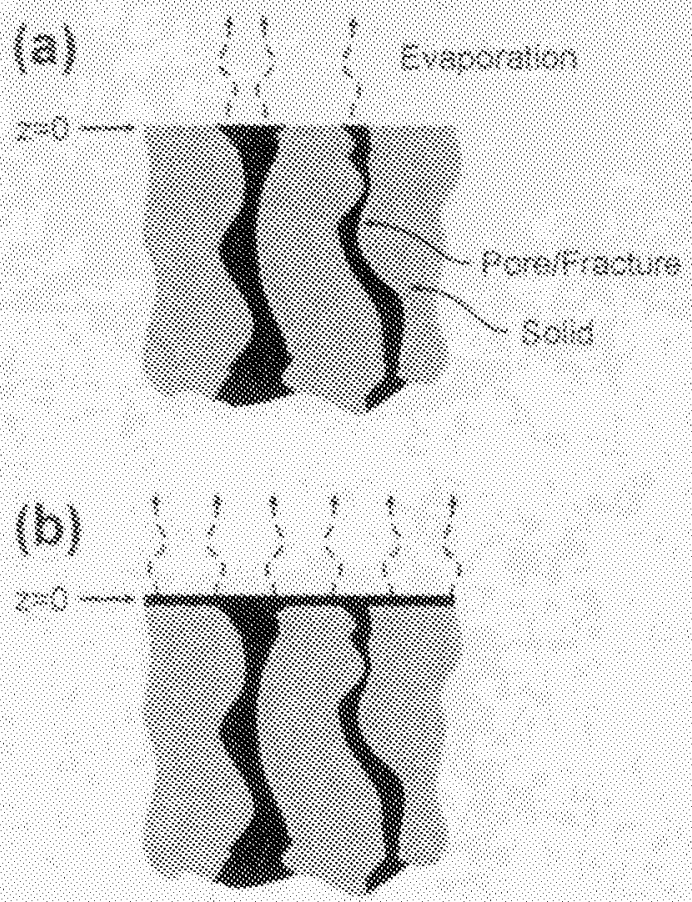

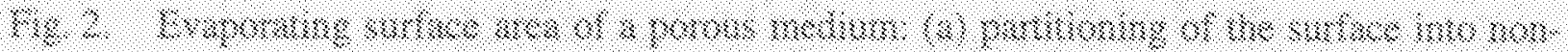

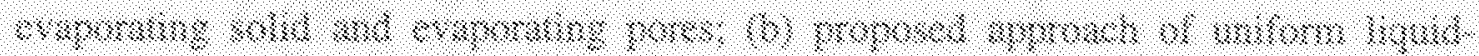

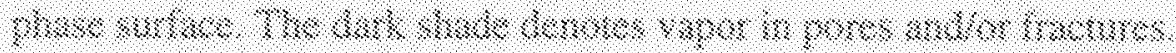




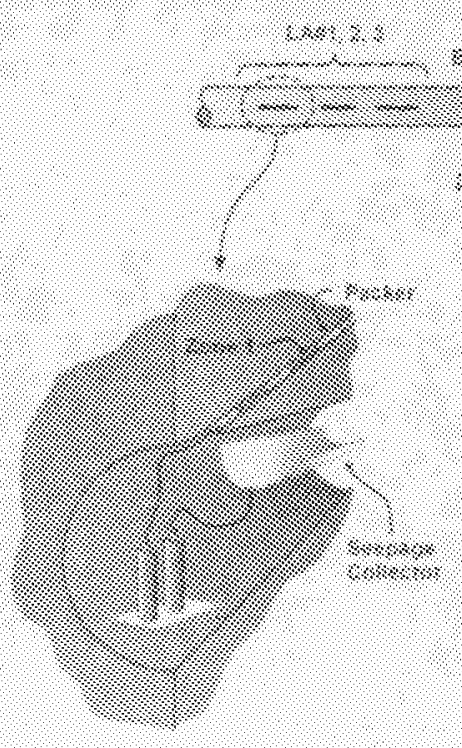

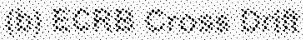

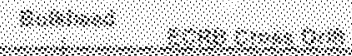

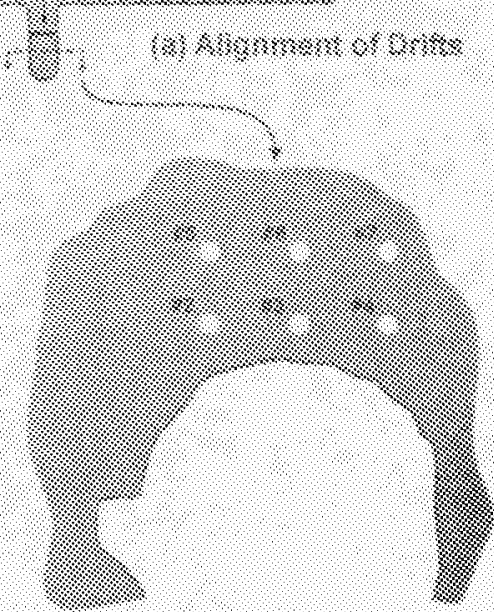

$1,8,18 \%$

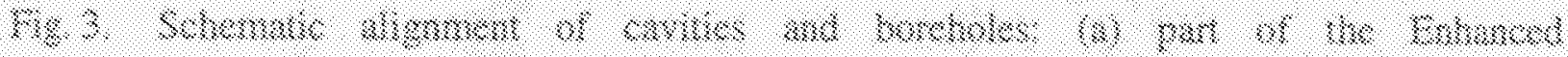

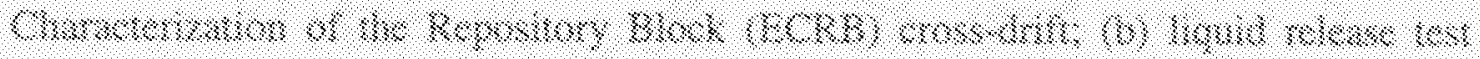

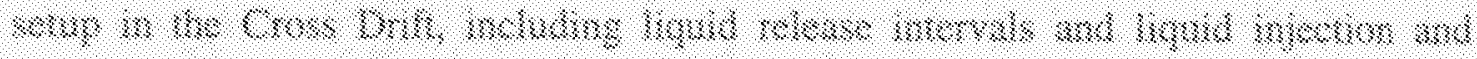

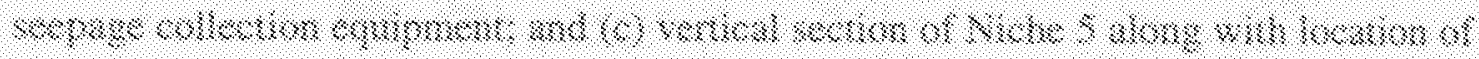

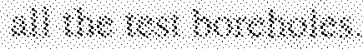




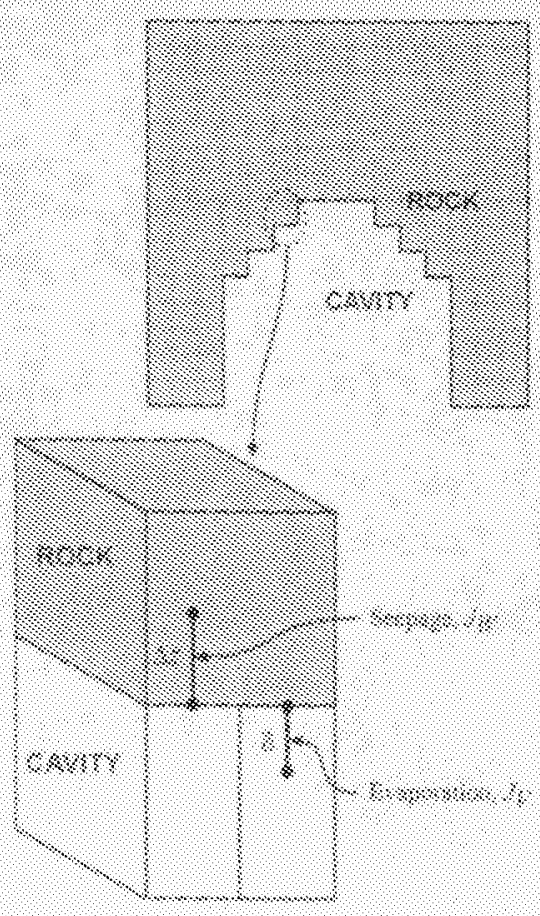

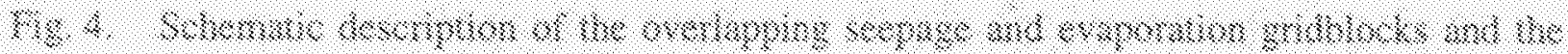

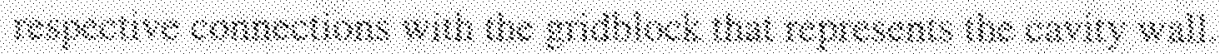



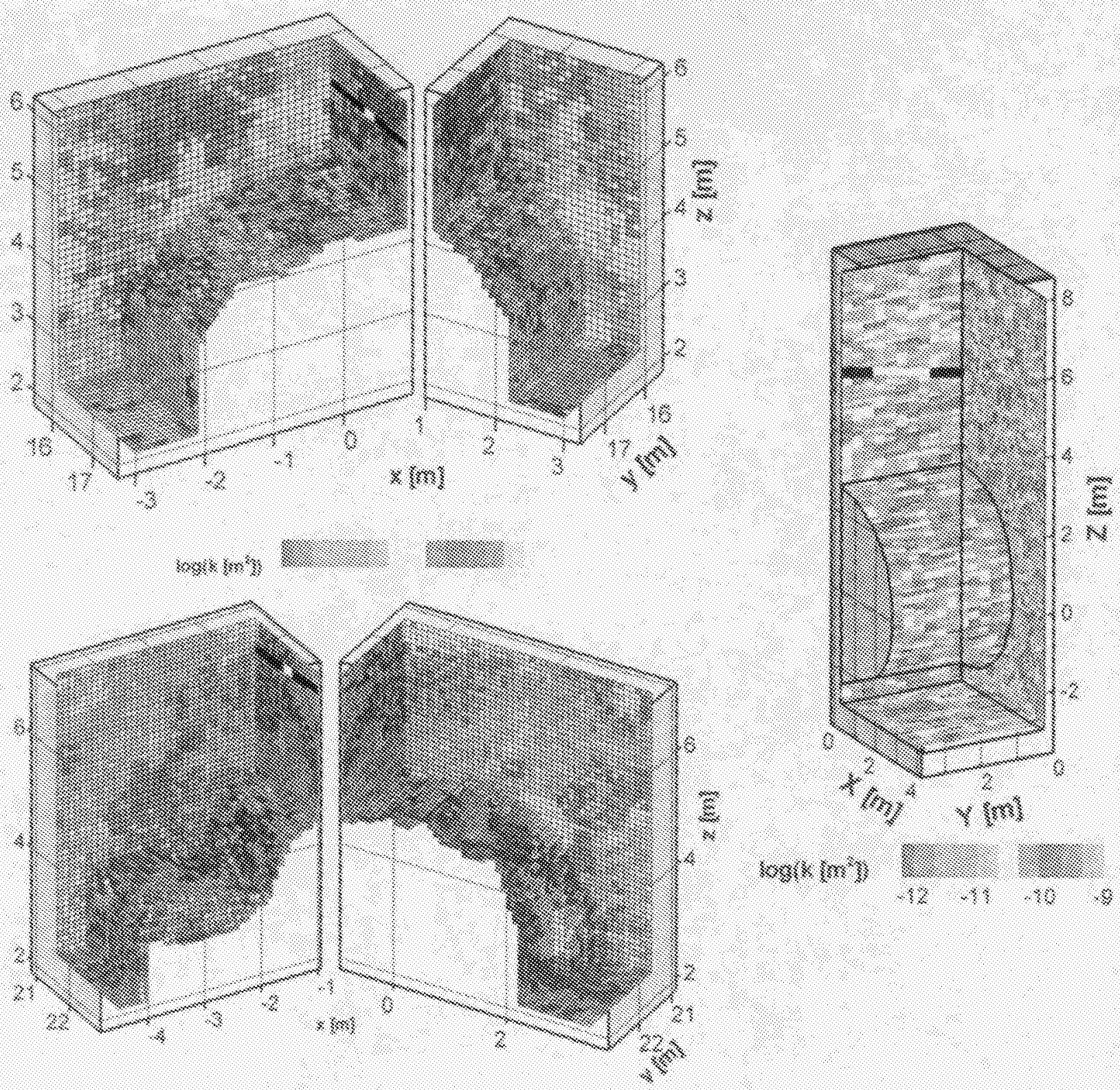

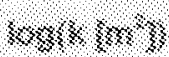

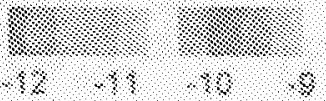

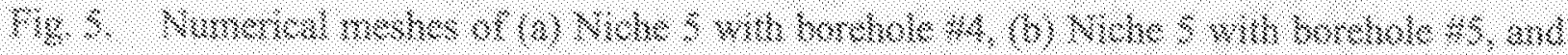

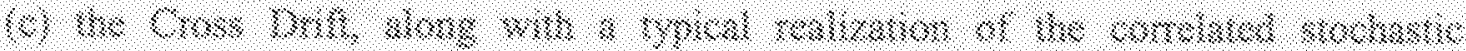

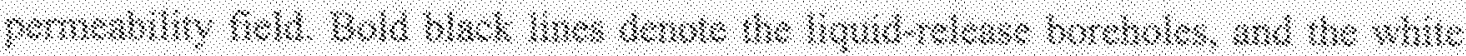

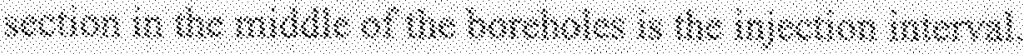


instide Niche 8
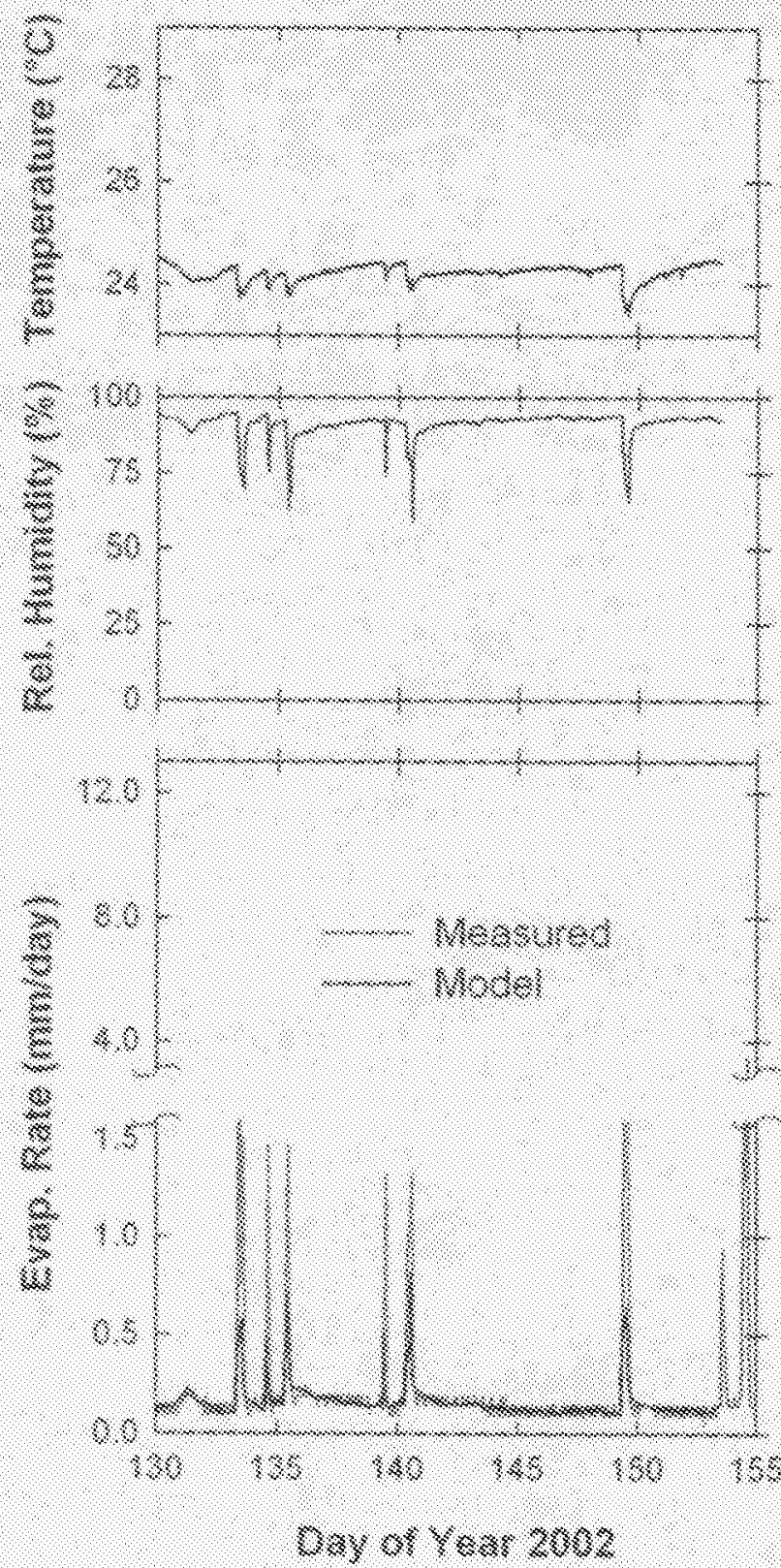

O)
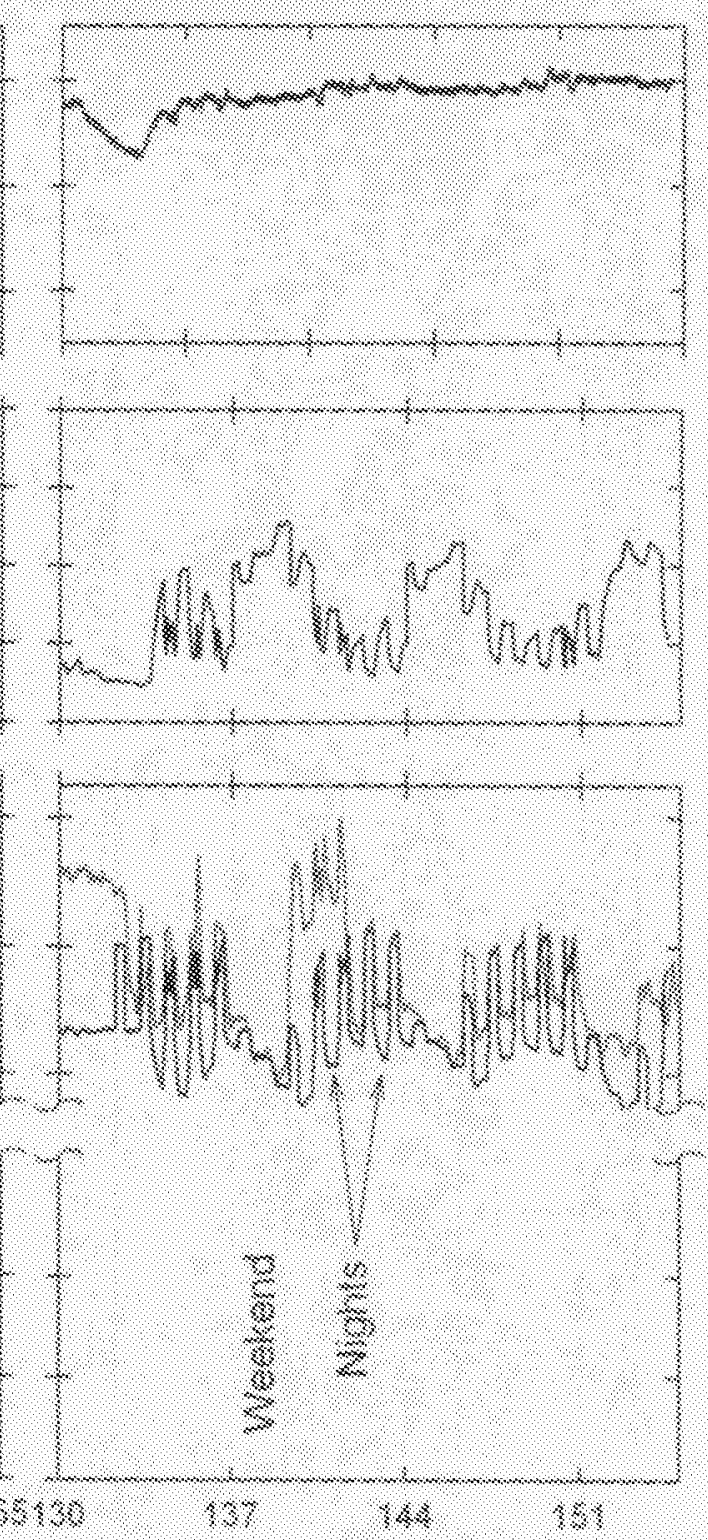

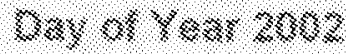

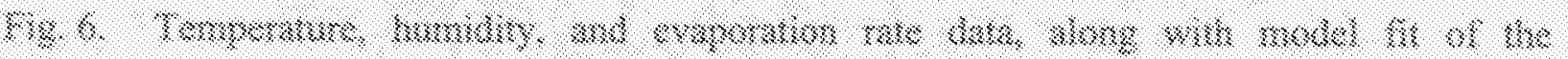

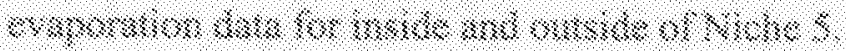


Whome 5. Doreho: 44

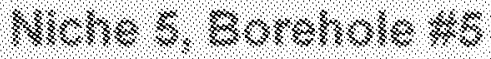
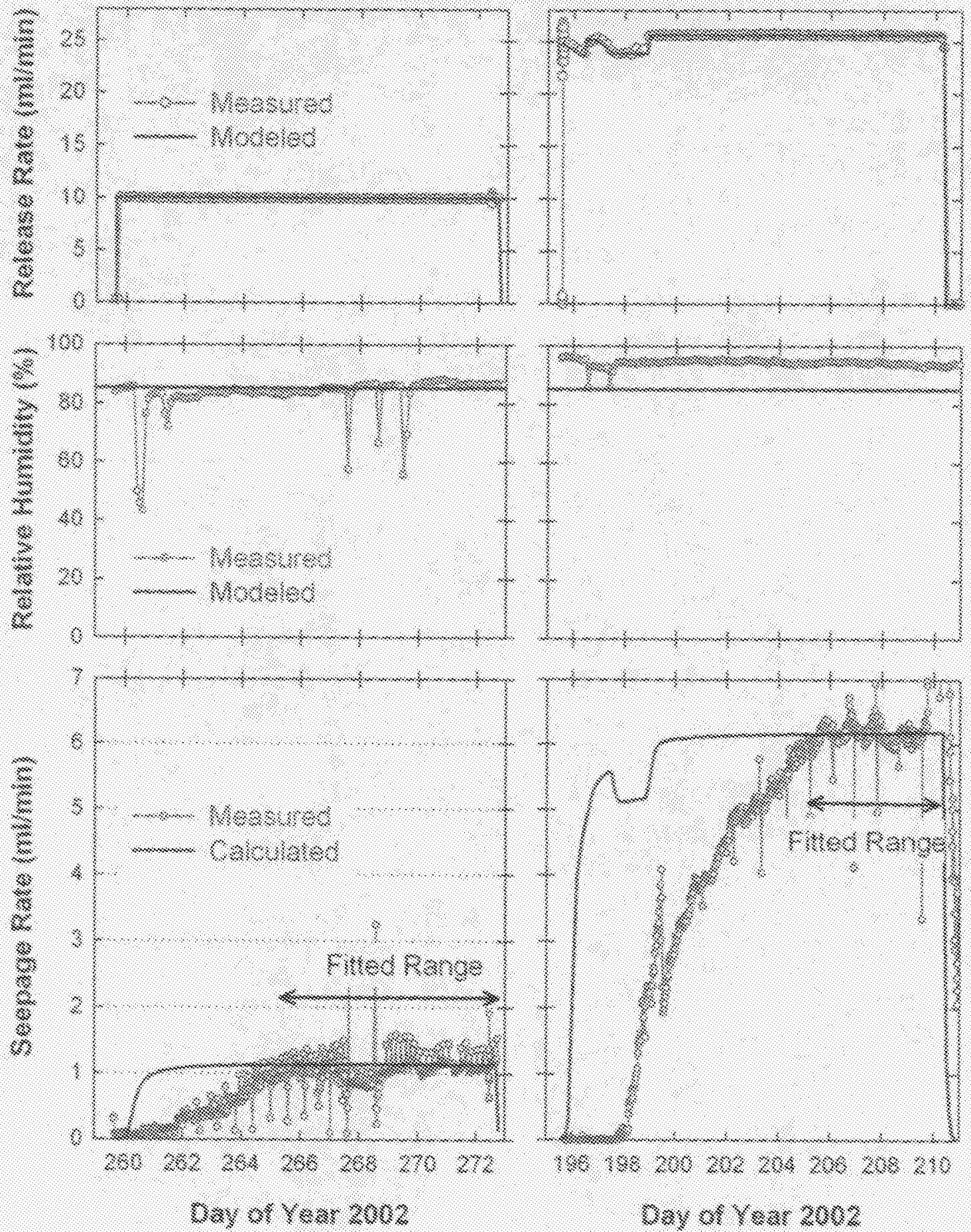

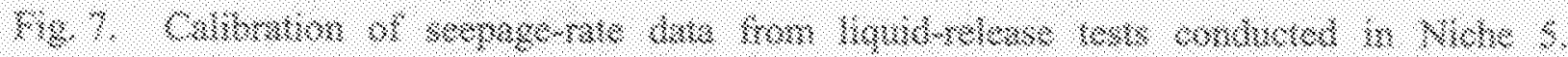

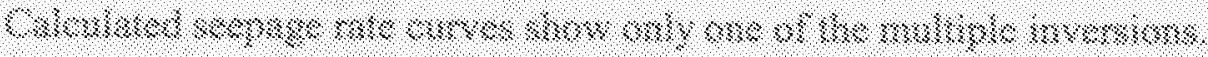




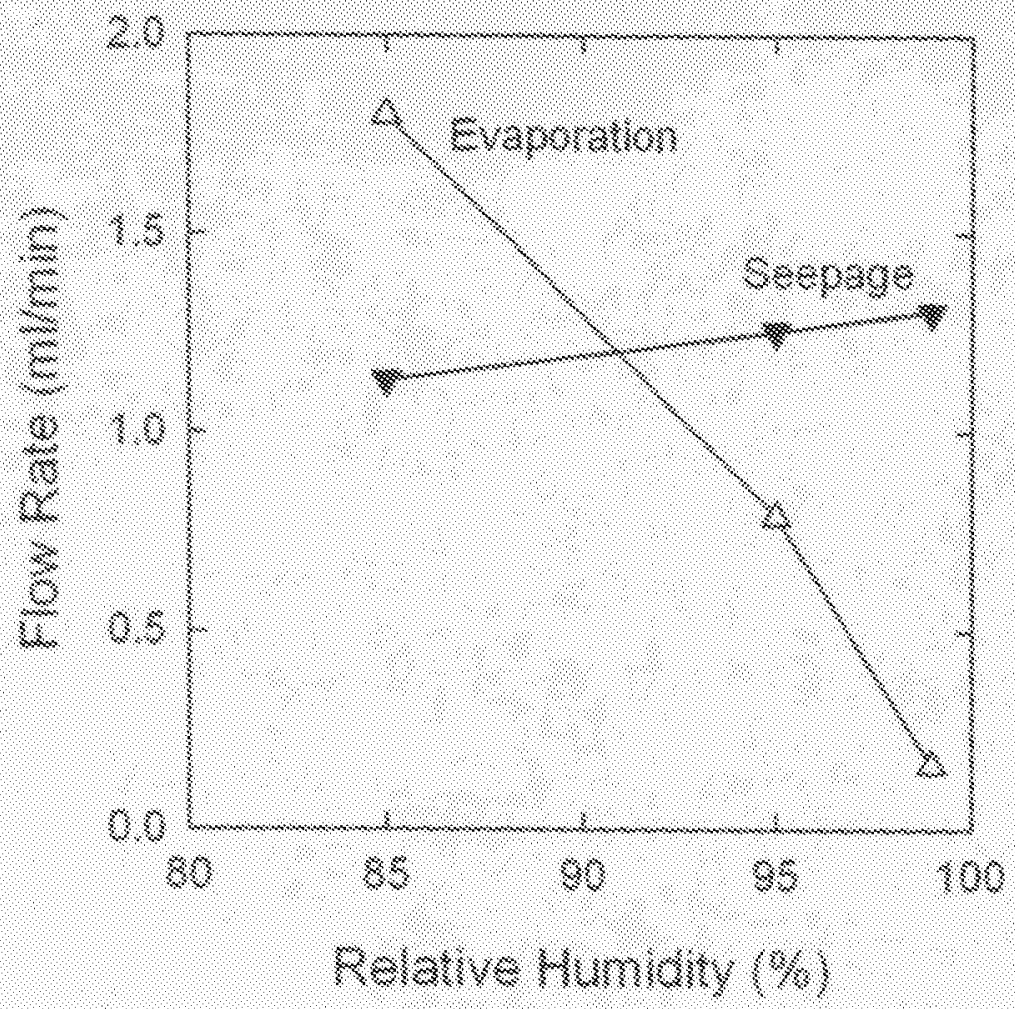

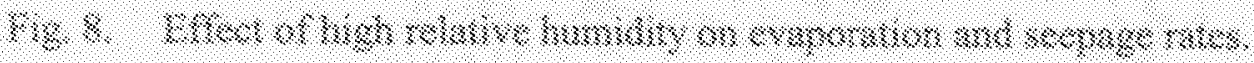


$4.4 * 2$ Z Zone 2
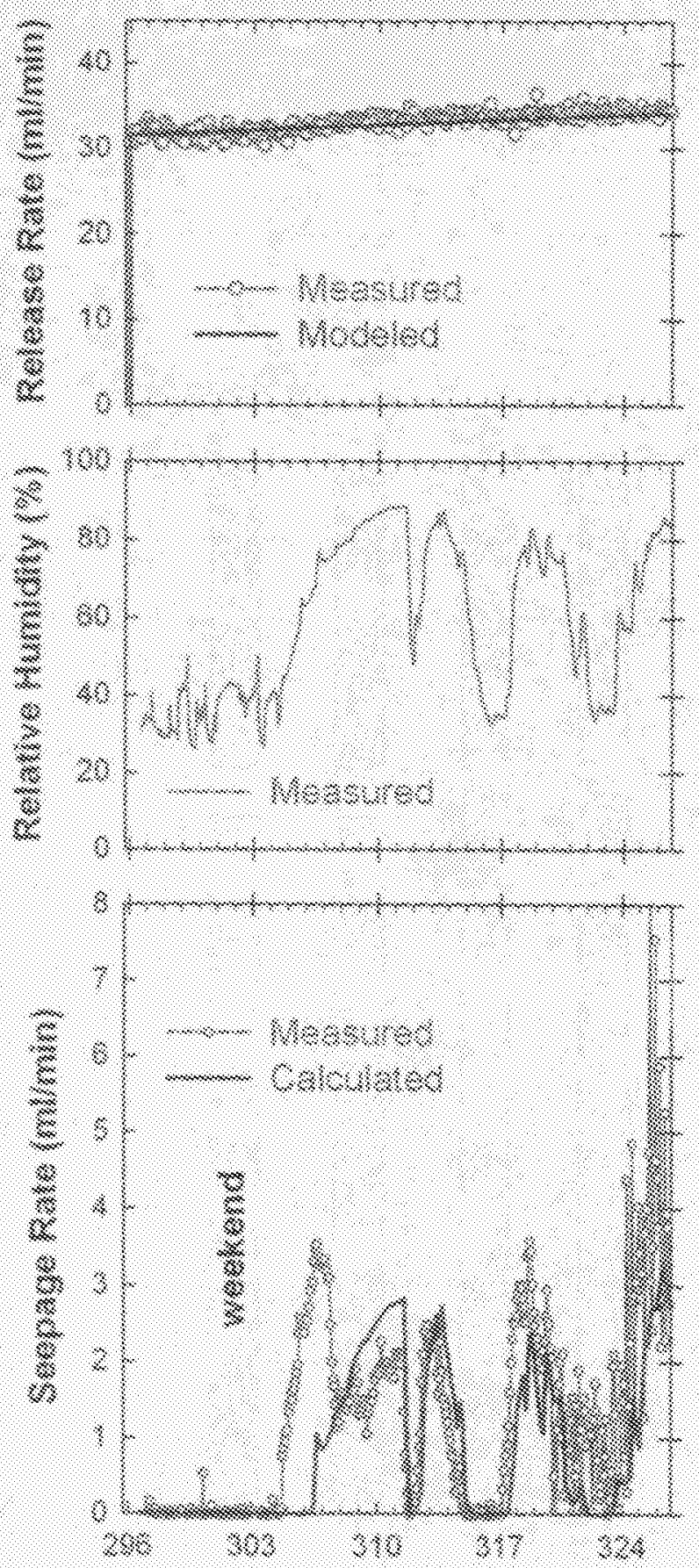

Domy of $Y$ sear 200

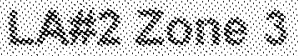
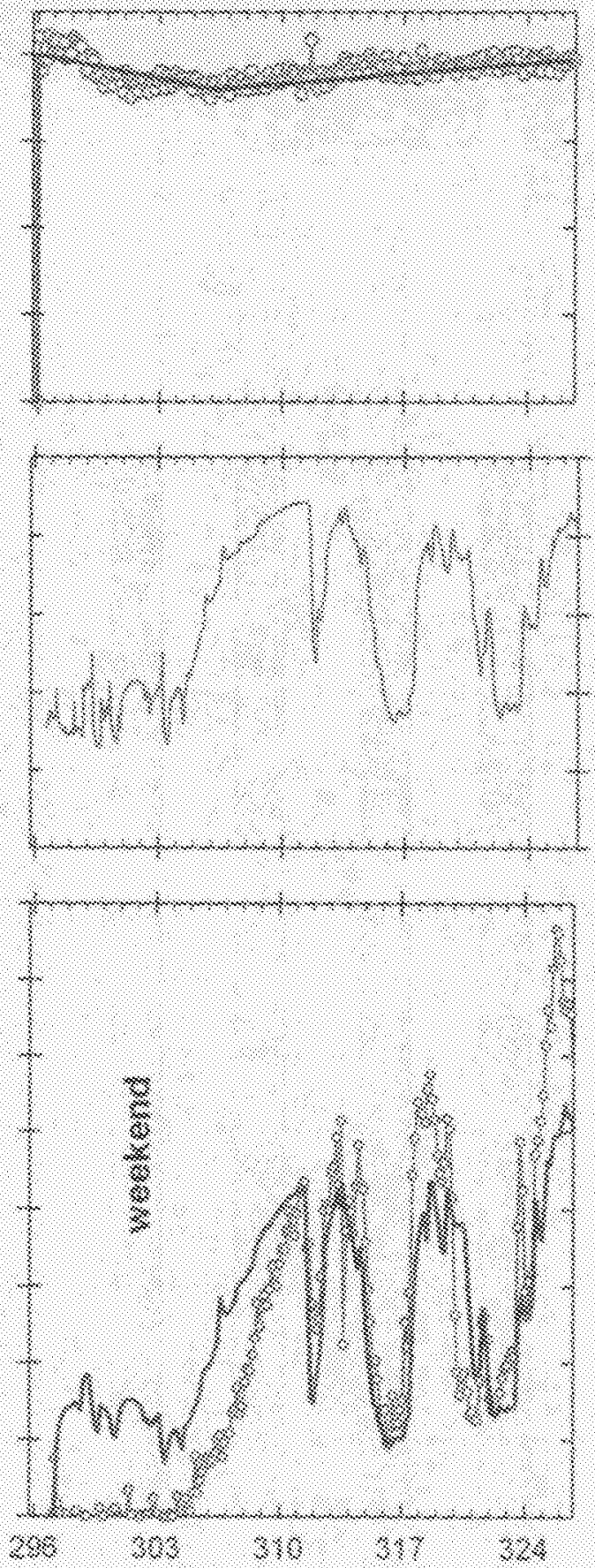

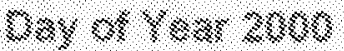

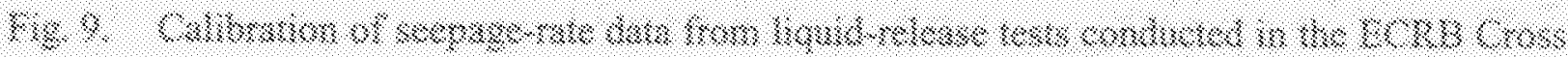

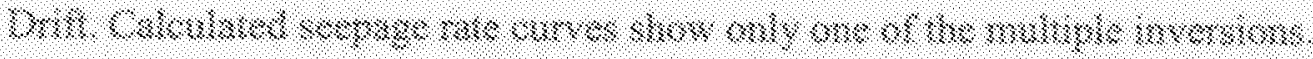




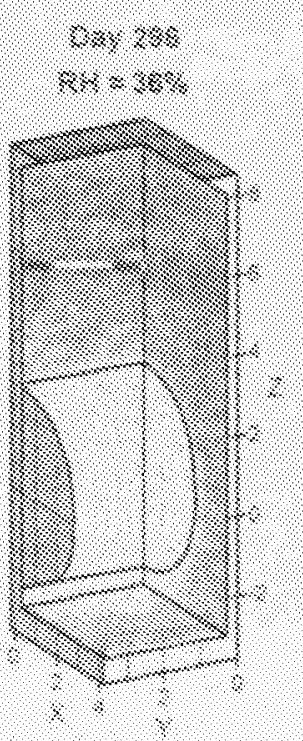

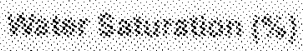

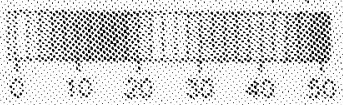

(2. $1.18 \%$

(1): $.1 .8 \%$

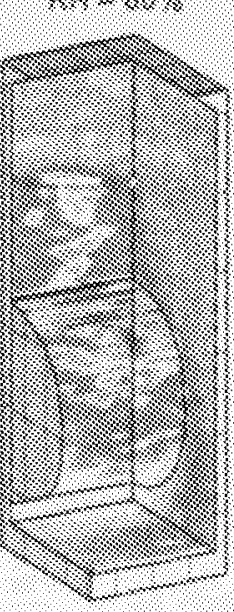

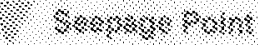

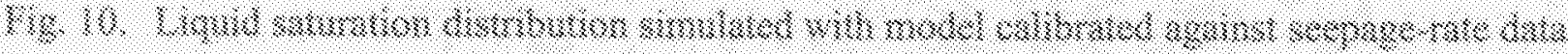

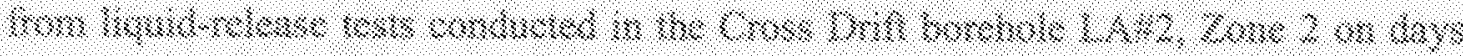

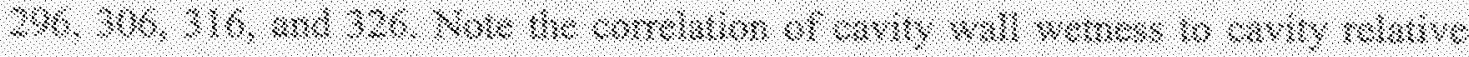
$30303: 30 \%: 3$ 


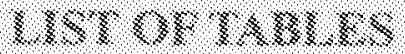

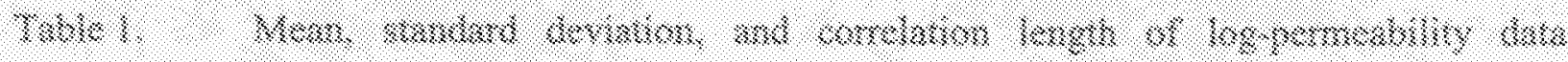

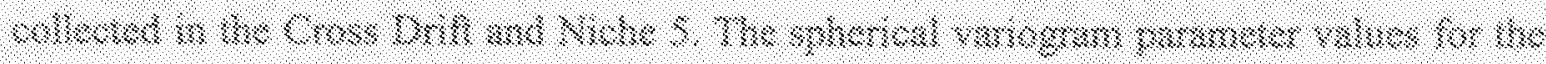
का: $40 m$ (3)

\begin{tabular}{|c|c|c|c|c|c|c|}
\hline \multirow[b]{2}{*}{ 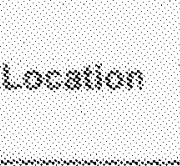 } & \multirow[b]{2}{*}{88} & \multirow{2}{*}{ 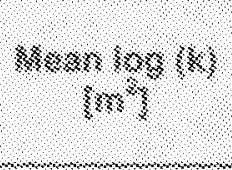 } & \multirow{2}{*}{ 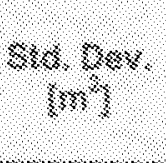 } & \multicolumn{3}{|c|}{ 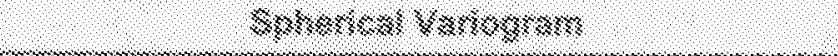 } \\
\hline & & & & 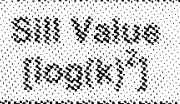 & 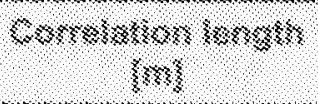 & 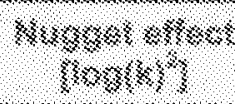 \\
\hline 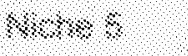 & $8 \%$ & 14048 & 3 & $98 \%$ & 484 & 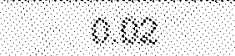 \\
\hline$\leftrightarrow \leftrightarrow \infty$ & 8 & $.401 \%$ & 323 & 48 & $4 \%$ & ? \\
\hline
\end{tabular}

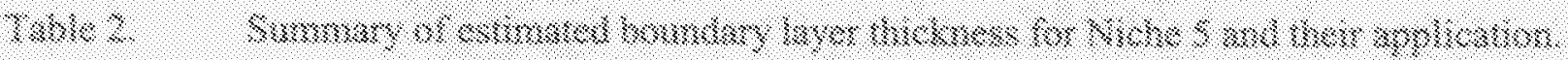

\begin{tabular}{|c|c|c|}
\hline 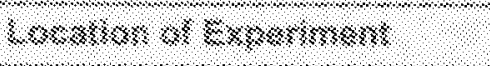 & $84: 00: 3\}$ & :3: \\
\hline 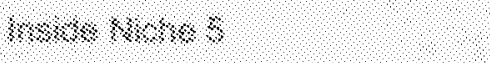 & 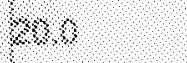 & $1,8: 2: 1: 0$ \\
\hline 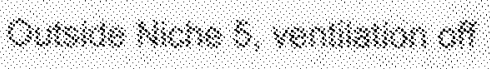 & 18 & 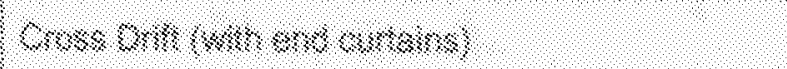 \\
\hline 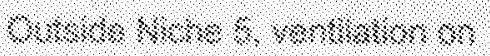 & 80 & $800640 \% 8 \%$ \\
\hline
\end{tabular}




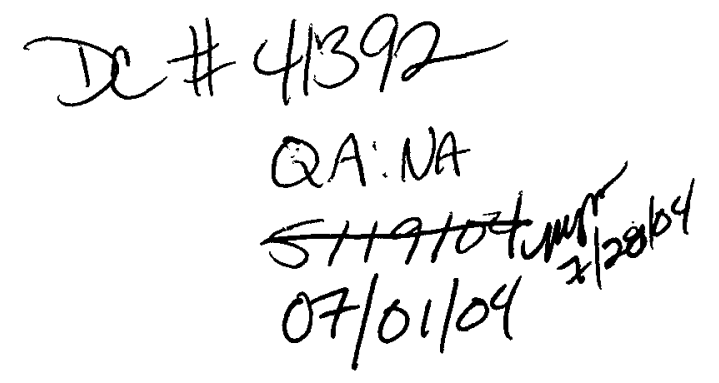

MOL . 20040828.0220

\section{Modeling Coupled Evaporation and Seepage in Ventilated Cavities}

T. A.Ghezzehei*, R. C. Trautz, S. Finsterle, P. J. Cook, and C. F. Ahlers ${ }^{1}$

Earth Sciences Division

Lawrence Berkeley National Laboratory

University of California

MS 90R1116, 1 Cyclotron Rd.,

Berkeley, CA 94720-8126

* Corresponding author, Tel (510) 486-5688, Fax (510) 486-5686, Email TAGhezzehei@lbl.gov

${ }^{1}$ Now at LFR Levine Fricke, Costa Mesa, California 


\section{ABSTRACT}

Cavities excavated in unsaturated geological formations are important to activities such as nuclear waste disposal and mining. Such cavities provide a unique setting for simultaneous

4 occurrence of seepage and evaporation. Previously, inverse numerical modeling of field liquid-

5 release tests and associated seepage into cavities were used to provide seepage-related large-

6 scale formation properties by ignoring the impact of evaporation. The applicability of such

7 models was limited to the narrow range of ventilation conditions under which the models were

8 calibrated. The objective of this study was to alleviate this limitation by incorporating

9 evaporation into the seepage models. We modeled evaporation as an isothermal vapor diffusion

10 process. The semi-physical model accounts for the relative humidity, temperature, and

11 ventilation conditions of the cavities. The evaporation boundary layer thickness (BLT) over

12 which diffusion occurs was estimated by calibration against free-water evaporation data

13 collected inside the experimental cavities. The estimated values of BLT were 5 to $7 \mathrm{~mm}$ for the

14 open underground drifts and $20 \mathrm{~mm}$ for niches closed off by bulkheads. Compared to previous

15 models that neglected the effect of evaporation, this new approach showed significant

16 improvement in capturing seepage fluctuations into open cavities of low relative humidity. At

17 high relative-humidity values (greater than $85 \%$ ), the effect of evaporation on seepage was very

18 small. 


\section{INTRODUCTION}

20 Seepage of liquid water into cavities is an important phenomenon for subsurface activities such as mining and geologic disposal of nuclear wastes. A key factor affecting the long-term safety of the proposed nuclear waste repository at Yucca Mountain (YM), Nevada, is the seepage of liquid water into waste emplacement cavities. The rate, chemical composition, and spatial and temporal distributions of seepage are critical factors that determine corrosion of waste canisters, integrity of engineered barriers, and dissolution and mobilization of contaminants and their release to groundwater (Bodvarsson et al., 1999; Finsterle et al., 2003). In unsaturated formations, capillary

27 forces hold the pore water tightly in the formation and divert the flow around the cavity thereby 28 preventing seepage into the cavity - the barrier created by the capillary force is commonly

29 known as a "capillary barrier." Philip and co-workers (Knight et al., 1989; Philip, 1989a; Philip, 30 1989b; Philip et al., 1989a; Philip et al., 1989b) considered steady-state unsaturated flow around 31 capillary barriers and provided analytical solutions for the critical conditions that trigger seepage

32 into various idealized cavity geometries excavated in homogeneous formations. Detailed 33 numerical models have been used to study unsaturated flow in heterogeneous fractured media 34 and seepage into cavities of various geometries under transient conditions (e.g., Birkholzer et al., 35 1999; Finsterle, 2000; Finsterle and Trautz, 2001; Li and Tsang, 2003). Site-specific seepage 36 models for the proposed nuclear waste repository at Yucca Mountain, Nevada, were developed 37 by calibrating the effective seepage-related parameters against field seepage test data (Finsterle 38 et al., 2003).

39 Most of the previous numerical models assumed that liquid water leaking into a cavity drips 40 (seeps) immediately from the place of entry. The potential for evaporation to compete with 
41 seepage has been generally ignored, and its effect was lumped with the effective flow parameters

42 of the unsaturated medium (Finsterle et al., 2003). In calibration of the analytical model of Philip

43 et al. (1989b) against field seepage data, Trautz and Wang (2002) accounted for the effect of

44 evaporation by adjusting the field seepage data for evaporation. Because the data were obtained

45 from tests conducted in relatively humid cavities, the effect of evaporation on the calibrated

46 seepage-related parameter was not significant. However, recent field measurements of seepage

47 and free-water evaporation in ventilated cavities at Yucca Mountain have shown that seepage

48 rate is significantly influenced by evaporation. The foregoing discussions suggest that the

49 applicability of models that ignore evaporation is limited to similar humidity and temperature

50 conditions under which the calibrations are performed (Finsterle et al., 2003). Such models

51 cannot satisfactorily capture the seepage rate fluctuations when the seepage experiments are

52 conducted under variable humidity and ventilation conditions. More importantly, seepage models

53 that ignore evaporation, and that are calibrated against seepage data under ventilated and/or low

54 humidity conditions are not expected to perform well in predicting future (expected non-

55 ventilated and humid) seepage conditions. The preceding observations call for a calibrated

56 seepage model that reliably performs over a wide range of ventilation and humidity conditions.

57 The objective of this study is to improve the portability of calibrated seepage models by reducing

58 the impact of evaporation on the calibrated effective parameters. Thus, we propose to incorporate

59 evaporation from cavity walls into the existing seepage models by assuming a first-order 60 diffusion approximation. 
62 Fundamentally, evaporation is a two-step process. The first step involves transition from liquid

63 to vapor phase at the liquid-vapor interface (vaporization). The second step is the transport of

64 vapor from the high concentration area at the evaporating surface to the low concentration area

65 of the ambient air. Accurate modeling of these coupled processes is difficult for several reasons:

66 (1) the first step is an endothermic phenomenon, and the parameters that govern this process are

67 strongly temperature dependent; (2) the vapor concentration gradient in the boundary layer is

68 strongly influenced by the air flow regime; and (3) the air flow depends on, among other things,

69 the ambient wind velocity, local turbulence, and the roughness of the evaporating surface.

Ho (1997) and Or and Ghezzehei (2000) modeled evaporation from individual water

71 droplets attached to cavity ceilings, assuming constant temperature and humidity conditions. In

72 the present study, we extend these evaporation models to account for evaporation from a variably

73 saturated porous surface. In contrast to the positive capillary pressure on the surfaces of

74 evaporating droplets, the porous surface typically has negative or zero capillary pressure. The

75 evaporation model used in this study is coupled with flow in the porous medium and the relative

76 humidity of the ambient air could vary.

The formulation used herein capitalizes on the observed dependence of evaporation rate on cavity humidity and ventilation conditions, and the availability of high resolution time-series data of relative humidity, temperature and free-water evaporation rate (Trautz and Wang, 2002).

In the following subsections, we introduce an isothermal vapor diffusion model of

81 evaporation and define the problem domain and boundary conditions. This is followed by 
82 estimation of the evaporation model parameters using free-water evaporation data. Finally, a 83 remark on evaporation from a porous surface is provided.

\section{Isothermal Vapor Diffusion Model}

85 The development of the isothermal vapor diffusion model presented in this subsection closely follows that of Ho (1997) with the exception of the range of vapor pressure on the evaporating surface. In the study of Ho (1997) the vapor pressure on the surface of the evaporating pendent drops depends on the drop radius (positive capillary pressure), while in our study the vapor pressure on the surface of the porous medium depends on the capillary pressure of the liquid held in the pores (negative or zero capillary pressure). To simplify the first step of evaporation (vaporization) we assume the following: (1) the absorption of latent heat and its effect on the physical properties of the liquid-vapor interface are negligible; (2) the time-dependence of the vaporization process (e.g., Zhang and Wang, 2002; Zhang et al., 2001) is neglected; and (3) the vapor partial pressure of the interfacial air is assumed to be under thermodynamic equilibrium. At equilibrium, the air above a flat surface of pure water is considered saturated with vapor; its vapor pressure is denoted by $p_{\mathrm{s}}[\mathrm{Pa}]$. This saturation vapor pressure rises with temperature. In the temperature range of $-10^{\circ} \mathrm{C}$ to $50^{\circ} \mathrm{C}$, the saturation vapor pressure is related to interfacial temperature by (Murray, 1966):

$$
\ln p_{\mathrm{s}}=a \frac{T}{T+b}+c
$$
where $a=21.87, b=265.5^{\circ} \mathrm{C}$ and $c=6.41$ are constants, and $T\left[{ }^{\circ} \mathrm{C}\right]$ is the interfacial

101 temperature. For non-flat interfaces (such as capillary menisci) the actual interfacial vapor 102 pressure $p$ is related to the interfacial capillary potential by the classic Kelvin equation, 


$$
\ln \left(\frac{p}{p_{\mathrm{s}}}\right)=P_{C} \cdot \frac{M_{W}}{\rho_{W} R T}
$$

104 where $P_{C}[\mathrm{~Pa}]$ is the capillary pressure, $\rho_{W}=998 \mathrm{~kg} \mathrm{~m}^{-3}$ and $M_{W}=0.018 \mathrm{~kg} \mathrm{~mol}^{-1}$ are the 105 density and molecular mass of liquid water, respectively, and $R=8.3143 \mathrm{~J} \mathrm{~K}^{-1} \mathrm{~mol}^{-1}$ is the 106 universal gas constant. Note that the relative humidity of air is defined as the ratio of the actual 107 partial pressure $(p)$ to the saturated vapor pressure $\left(p_{s}\right)$

$$
h=p / p_{\mathrm{s}}
$$

109 The second step of evaporation, vapor removal from the interface, is modeled as a first-order 110 phenomena described by Fickian diffusion (Rohsenow and Choi, 1961). In one dimension and 111 under constant temperature, the vapor mass flux $\left(J_{V}\left[\mathrm{~kg} \mathrm{~m}^{-2} \mathrm{~s}^{-1}\right]\right)$ is given by

$$
\left.J_{V}\right|_{T}=-D_{V} \cdot \frac{\mathrm{d} C}{\mathrm{~d} z}
$$

113 where $D_{V}\left[\mathrm{~m}^{2} \sec ^{-1}\right]$ is the vapor diffusion coefficient, which is related to the ambient air 114 pressure ( $P[\mathrm{~Pa}])$ and air temperature $(T)$ by (Vargaftik, 1975)

$$
D_{V}=2.13 \times 10^{-5} \frac{10^{5}}{P}\left(\frac{T}{273.15}\right)^{1.8}
$$

116 and the vapor concentration $C\left[\mathrm{~kg} \mathrm{~m}^{-3}\right]$ is related to vapor pressure by

$$
C=\frac{M_{W}}{R T} \cdot p
$$

118 The error introduced by using the equimolal counterdiffusion equation (Eq. [4]) to represent 119 evaporation (vapor diffusion in stationary gas) is ignored. In the subsequent subsection, we 120 define the problem domain and develop the appropriate boundary conditions needed to solve the 121 vapor diffusion equation [4]. 


\section{Velocity and Concentration Boundary Layers}

123 In admitting diffusive flux as the primary mechanism for vapor removal from the evaporating

124 surface, we assume that airflow above the evaporating surface is fully developed and laminar, as

125 illustrated in Fig. 1a. The free-stream air velocity $\left(U^{\infty}\left[\mathrm{m} \mathrm{s}^{-1}\right)\right.$ is retarded in the vicinity of the 126 evaporating surface because of frictional resistance. The air velocity parallel to the evaporating 127 surface increases from $U=0$ at $z=0$ (no-slip) asymptotically to $U=U^{\infty}$ at a distance 128 sufficiently far away from the surface. For fully laminar flow conditions, the thickness of the 129 boundary layer $\left(\delta_{U}\right)$ of retarded velocity (defined as $U \leq 0.99 U^{\infty}$ ) is inversely proportional to the 130 square root of the free-stream velocity (Rohsenow and Choi, 1961):

$$
\delta_{U} \propto 1 / \sqrt{U^{\infty}}
$$

132 Because the equations that describe laminar air flow parallel to a flat surface and diffusion from 133 a flat surface are analogous (Rohsenow and Choi, 1961), a similar notion of concentration 134 boundary layer holds near the evaporating surface. The vapor concentration profile is illustrated 135 in Fig. 1b. The vapor concentration decreases from an equilibrium value $\left(C=C^{0}\right)$ at $z=0$ to a 136 value determined by the free-stream humidity at sufficiently far distance. The concentration 137 boundary layer thickness $\left(\delta_{C}\right)$ is related to the velocity boundary layer thickness by the Schmidt 138 number,

$$
S c=\frac{\delta_{C}}{\delta_{U}}=\frac{\mu_{a}}{\rho_{a} \cdot D_{V}}
$$
where $\mu_{a}[\mathrm{~Pa} \mathrm{~s}]$ and $\rho_{a}$ are the viscosity and density of air, respectively. At $20{ }^{\circ} \mathrm{C}$ and 1 atm

141 pressure, the Schmidt number is approximately unity. In the remainder of this paper the 142 subscripts in the boundary layer thickness are dropped and $\delta=\delta_{U}=\delta_{C}$ [m]. It is evident from [7] 
and [8] that the concentration $\operatorname{BLT}(\delta)$ is inversely related to the square root of the free-stream

velocity $\left(U^{\infty}\right)$ and can serve as a direct measure of the cavity ventilation condition. In a

subsequent subsection, estimation of the BLT will provide further elaboration on the dependence

146 of $\delta$ on ventilation conditions.

\section{Boundary Conditions}

148 The domain of the vapor diffusion equation [4] is the concentration boundary layer introduced in

149 the preceding subsection. The boundary condition on [4] corresponding to the equilibrium vapor concentration at the evaporating surface $(z=0)$ is given by (using [2] and [6]):

$$
C=C^{0}=\frac{M_{W}}{R T} p_{s} \exp \left[\frac{P_{C}}{\rho_{W}} \frac{M_{W}}{R T}\right]
$$

The second boundary condition is at the border of the concentration boundary layer $z=\delta$, where the vapor concentration is defined by the relative humidity $(h)$ of the ambient air:

$$
C=C^{\infty}=\frac{M_{W}}{R T} p_{s} h
$$

If the boundary conditions change slowly, the evaporation rate can be considered to be at steady state and the concentration gradient $\mathbf{d} C / \mathbf{d} z$ is constant throughout the boundary layer. Then, the steady-state vapor diffusion equation [4] under isothermal conditions is simplified to

$$
J_{V}=D_{V} \cdot \frac{C^{0}-C^{\infty}}{\delta}
$$

Note that the ratio $\delta / D_{V}$ is commonly referred to as diffusional resistance and [11] is essentially

Dalton's equation as used by Penman (1948). The isothermal vapor diffusion equation [11] is considered valid for modeling evaporation from cavity surfaces and free water. Fujimaki and Inoue (2003) found [11] (also known as the bulk transfer equation) to be valid in laboratory 
163 evaporation experiments in which the ambient air velocity was on the order of $1 \mathrm{~m} / \mathrm{s}$. All the

164 variables of this model are directly related to physical conditions in the cavity, and all of them,

165 except $\delta$, can be independently determined from measured quantities. The boundary-layer

166 thickness $(\delta)$ can be estimated by calibrating [11] against free water evaporation data, as

167 discussed in the next subsection.

\section{Estimation of the Boundary-Layer Thickness}

Apart from the capillary pressure at the evaporating surface, evaporation from free water and that

from a wet porous surface are thus far assumed to be identical processes. Therefore, a controlled evaporation experiment from a still-water surface can be used to estimate the vapor concentration boundary layer thickness, which is also applicable to evaporation from wet cavity surfaces at similar ventilation conditions. Upon substitution of [1], [5], [9], and [10] in [11], and noting that the capillary pressure of the free-water surface is $P_{C}=0$, we arrive at a free-water evaporation equation,

$$
J_{V}=2.13 \times 10^{-5} \frac{10^{5}}{P}\left(\frac{T}{273.15}\right)^{1.8} \cdot \exp \left(a \frac{T}{T+b}+c\right) \cdot \frac{M_{W}}{R T} \frac{1-h}{\delta}
$$

177 According to the isothermal assumption, $T$ denotes the temperature of the evaporating surface and the surrounding air. Assuming the change in conditions that affect evaporation rate is slow compared to the time it takes to reach steady-state evaporation, [12] can be fitted to time-series data of evaporation rate data measured at known temperature, pressure, and relative humidity conditions. The best-fit $\delta$ represents the boundary-layer thickness at the prevailing ventilation

182 condition. However, it should also be noted that uncertainties associated with the assumed 183 simplifications (including isothermal conditions, flat evaporating surface, and laminar airflow) 
are lumped in this parameter. Thus, the boundary-layer thickness should be considered an

185 effective parameter.

\section{Evaporation from Porous Surface}

187 The surface of an unsaturated porous medium typically consists of solid (matrix of the medium)

188 and pore/fracture (liquid and gas) components, rendering the evaporating surface heterogeneous

189 with respect to vapor concentration, as illustrated in Fig. 2a. During seepage, however, cavity

190 ceilings are usually covered with liquid films (e.g., Trautz and Wang, 2001), and the vapor

191 concentration could be considered locally homogeneous. For simplicity, we extend this

192 assumption of locally uniform distribution of vapor concentration to the entire cavity Fig. 2 b.

193 The vapor concentration at any given location on the cavity is assumed to be at capillary

194 equilibrium with the pores and fractures of the porous medium. The datum $z=0$ for the vapor

195 diffusion is set on the surface of the cavity (as illustrated in Fig. 2b). Although this assumption is

196 likely to fail at very low saturations (when the liquid is scattered in a few fine pores and

197 fractures) it is expected to be of marginal consequence because the evaporation rate under such

198 conditions is very low. Moreover, as indicated in the preceding subsection, uncertainties

199 regarding the geometry of the evaporating pan are lumped in the calibrated boundary layer

200 thickness. It should be noted that an unknown error is introduced when applying this boundary

201 layer thickness value to evaporation from rough cavity surfaces that have significantly different

202 geometry from the relatively flat free water surfaces.

204 In a cavity constructed in unsaturated formations, the flow velocity of water in the rock is usually 205 stagnated near the crown, resulting in an elevated moisture content in this region (Philip et al., 
1989b). Unlike evaporation from a ground surface, where infiltration opposes the evaporation

207 flux, the condition in cavities is favorable for simultaneous occurrence of evaporation and seepage. Field tests that exhibit simultaneous evaporation and seepage are described below. After

field-test descriptions, we present a brief description of seepage modeling using the numerical

210 simulators TOUGH2 (Pruess et al., 1999) and iTOUGH2 (Finsterle, 1999) and discuss

211 implementation of evaporation in these models.

\section{Field Tests}

213 The data reported in this paper were obtained from field tests and measurements conducted at the

214 proposed nuclear waste repository at Yucca Mountain currently under investigation by the US

215 Department of Energy (DOE). Air-injection tests were conducted to characterize the

216 permeability and small-scale heterogeneities of the formation, and liquid-release tests were

217 performed to study seepage phenomena. Relative humidity, temperature, and free-water

218 evaporation were monitored at the test site to assess the evaporation conditions. Detailed

219 description of the site and tests conducted at the site are provided elsewhere (Birkholzer et al.,

220 1999; Bodvarsson et al., 1999; Finsterle and Trautz, 2001; Finsterle et al., 2003; Trautz and

221 Wang, 2001; Trautz and Wang, 2002; Wang et al., 1999). This study is concerned with the lower

222 lithophysal welded tuff unit at Yucca Mountain, in which about $80 \%$ of the proposed repository

223 is expected to reside. This unit contains many small fractures (less than $1 \mathrm{~m} \mathrm{long}$ ) and is

224 interspersed with numerous lithophysal cavities $(0.15 \mathrm{~m}-1 \mathrm{~m}$ in diameter).

225 In the lower lithophysal unit, an 800-m long drift (5 $\mathrm{m}$ in diameter) for enhanced characterization

226 of the repository block (ECRB) was excavated. Liquid-release and air-injection tests were

227 systematically conducted in this ECRB Cross Drift along boreholes drilled into the ceiling of the

228 Cross Drift at regular intervals. Similar tests were conducted in a short (approximately $15 \mathrm{~m}$ 
long) drift excavated off the Cross Drift (niches). Schematic alignment of the cavities is shown in

230 Fig. 3a. This paper is concerned with tests conducted at a Cross-Drift borehole designated as

231 LA\#2 (Fig. 3b) and a short drift known as Niche 5 (Fig. 3c). The tests and measurements

232 conducted in the Cross Drift and Niche 5 are briefly described below. In the remainder of this

233 paper, drifts and niches are referred to by the generic term cavity.

\section{Air-injection tests}

235 The purpose of the air-injection tests was to estimate absolute permeability of the formation as a

236 basis for the stochastic generation of heterogeneous permeability fields. Short sections of the

237 boreholes $(0.3 \mathrm{~m}$ in Niche $5,1.8 \mathrm{~m}$ in Cross Drift) were isolated using an inflatable packer 238 system, and compressed air was injected. Air injection was terminated when steady-state

239 pressure was reached. Air-permeability values were derived from the steady-state pressure data 240 according to an analytical solution of LeCain (1995). Permeabilities determined from air241 injection tests were considered representative of the absolute permeability of the test interval 242 because the air-injection tests were conducted in a network of essentially dry fractures before the 243 liquid-release tests were conducted. Therefore, no empirical relative permeability function is 244 needed to translate air conductivity into absolute permeability.

\section{Liquid-release Tests}

246 Liquid-release tests were conducted in boreholes drilled above cavities to evaluate seepage into 247 waste emplacement drifts. The alignment of the boreholes and test intervals are schematically 248 shown in Fig. 3. The liquid-release boreholes in the Cross Drift were approximately $20 \mathrm{~m}$ long, 249 drilled into the ceiling of the Cross Drift at a nominal inclination of $15^{\circ}$ from the horizontal. 250 Liquid-release data from a borehole designated as LA\#2 were used in this study. The borehole 251 was partitioned into three zones (designated as Zone 1, Zone 2, and Zone 3) available for liquid 
252 release testing. The distances from the middle of the liquid-release zones to the drift crown were

$2531.58 \mathrm{~m}, 2.84 \mathrm{~m}$, and $4.10 \mathrm{~m}$ for Zone 1, Zone 2, and Zone 3, respectively. The liquid-release

254 boreholes in Niche 5 were near horizontal. In this study, we used data from tests (boreholes \#4 255 and \#5) that lasted long enough to achieve nearly steady-state seepage. The liquid-release tests 256 were performed by injecting water into a test interval isolated by inflated rubber packers. Water 257 that seeped into the cavities was captured and measured using automated recording devices.

258 Relative Humidity and Temperature Measurements

259 The Cross Drift was actively ventilated during regular working hours, thus the relative humidity 260 of the cavity was usually low. Because the primary objective of the liquid-release tests was to 261 calibrate the seepage models, minimization of evaporation was an essential component of the 262 tests. To mitigate the effect of evaporation in the seepage process, the seepage collection interval 263 of the cross-drift was guarded using curtains on both ends. The relative humidity in Niche 5 was 264 relatively high because it was isolated from the actively ventilated Cross Drift by a bulkhead. To 265 aid in the estimation of evaporation during the liquid-release tests, the relative humidity and 266 temperature of the air inside and outside of the curtains (for the Cross Drift) and in front of and 267 behind the bulkhead (for Niche 5) were monitored using Vaisala HMP45C probes (RH 268 measurement range $0-100 \%$ non condensing, accuracy at $20{ }^{\circ} \mathrm{C} \pm 2 \%$ for $0-90 \% \mathrm{RH}$ and $\pm 3 \%$ for 269 90-100\% RH; Temperature measurement range $-40{ }^{\circ} \mathrm{C}$ to $60{ }^{\circ} \mathrm{C}$, accuracy $\pm 1 \%$ ). Up to 11 270 sensors were distributed in different parts of the cavities and averaged values are reported here.

271 The evaporation rate from still water was measured by monitoring the level (mass) of water in 272 evaporation pans placed within the space enclosed by the seepage capture tray and end curtains 273 (for the Cross Drift tests) and behind the bulkhead (for Niche 5). 


\section{TOUGH2/iTOUGH2 Seepage Model}

275 A detailed description of the numerical models developed for flow in a fractured formation 276 around a cavity and associated seepage into the cavity using TOUGH2/iTOUGH is given by 277 Finsterle et al. (2003). A summary of the seepage model follows.

278 We are concerned with the effective seepage behavior on the scale of a drift segment rather than 279 with individual seepage events from specific dripping locations. Thus, the unsaturated flow 280 through the densely fractured formation and seepage into the cavities is represented by a 281 simplified, heterogeneous continuum model. The matrix is not explicitly represented in this 282 single-continuum model because its contributions to flow and seepage are expected to be small. 283 The effects the matrix and microfractures are accounted for through the estimation of effective 284 parameters (Finsterle et al., 2003).

285 The TOUGH2 module we used (EOS9) is an integral finite difference simulator that represents 286 unsaturated flow at the scale of individual gridblocks by Richards' equation (Bear, 1972; Pruess 287 et al., 1999)

$$
\phi \rho \frac{\partial}{\partial t} S_{e}=\operatorname{div}\left[k \frac{\rho}{\mu} \nabla\left(P_{C}+\rho g z\right)\right]
$$

where $\rho$ and $\mu$ are the density and viscosity of water, $\phi$ is the porosity, and the effective saturation, $S_{e}$, is defined as $S_{e}=\left(S-S_{l r}\right) /\left(1-S_{l r}\right)$, with $S_{l r}$ being the residual liquid saturation.

291 The appropriateness of using this continuum approach to simulate water flow through 292 unsaturated fractured rock was shown by Finsterle (2000). The effective permeability $\left(k\left[\mathrm{~m}^{2}\right]\right)$ 293 and capillary pressure $\left(P_{C}\right)$ are functions of liquid saturation as given by van Genuchten's 294 models (1980) 


$$
\begin{gathered}
k=k_{a} S_{e}^{1 / 2}\left[1-\left(1-S_{e}^{1 / m}\right)^{m}\right]^{2} \\
P_{C}=-\frac{1}{\alpha}\left[S_{e}^{-1 / m}-1\right]^{1-m}
\end{gathered}
$$

where $k_{a}$ is the absolute permeability, and $1 / \alpha[\mathrm{Pa}]$ and $m$ are fitting parameters with $\alpha>0$ and $0<m<1$. While the $k_{a}$ values were considered spatially heterogeneous, the $1 / \alpha, m$, and $S_{l r}$ parameters were assumed to be homogeneous for a given test bed (Finsterle et al., 2003). The absolute permeability, $k_{a}$, was derived from the air-injection tests. The van Genuchten $m$ parameter and the residual saturation were taken to be $m=0.608$ and $S_{l r}=0.01$, respectively 302 (Finsterle et al., 2003). The van Genuchten capillary strength parameter $1 / \alpha$ was estimated 303 through inverse modeling. In the numerical seepage model, the condition for seepage is determined by the total water-potential gradient at the connection between the porous medium and the cavity, as depicted in Fig. 4. The mass flux of seepage water $J_{W}\left[\mathrm{~kg} \mathrm{~m}^{-2} \mathrm{~s}^{-1}\right]$ along the

306 connection between the porous medium and the cavity is given by

$$
J_{W}=-k \frac{\rho}{\mu} \frac{\Delta P+\rho g \Delta z}{\Delta z}
$$

308 where $\Delta P$ denotes the capillary pressure difference across the distance between the last

309 formation node and the cavity node $\Delta z=0.05 \mathrm{~m}$. From [16], and assuming that the capillary

310 pressure in the opening is zero, it follows that downward seepage $\left(J_{w}>0\right)$ occurs only when the

311 following condition is satisfied:

$$
-P_{C}^{*}>\rho g \Delta z
$$

313 where $P_{C}{ }^{*}$ is the threshold capillary pressure at the last node adjacent to the opening. The critical

314 capillary pressure $P_{C}{ }^{*}=-\rho g \Delta z$ depends on the grid size or nodal distance of the numerical 
315 model. Thus, the applicability of calibrated $1 / \alpha$ parameter is limited to the specific numerical

316 scheme used during calibration (Finsterle et al., 2003). According to [17], the cavity surface does

317 not need to be fully saturated for seepage to commence as in the case of unfractured

318 homogeneous porous media (Philip et al., 1989b).

\section{Implementation of Evaporation in TOUGH2}

320 While seepage occurs only when the critical condition given in [17] is satisfied, vapor flow

321 from/to cavity walls to/from cavity air occurs as long as there is vapor pressure disequilibrium

322 between them. Coupling of the seepage and evaporation processes is illustrated in Fig. 4. Mass-

323 transfer rate of water, including seepage, is represented in TOUGH2 by equations similar to [16],

324 where the driving force is pressure gradient. To incorporate evaporation into the existing

325 iTOUGH2 model without significant changes to the governing flow equations, the concentration-

326 gradient-dependent diffusion equation [11] was rewritten in the form of equation [16]. Thus, for

327 the purpose of evaporation from the cavity walls, the nodal distance $\Delta z$ is equivalent with the

328 vapor concentration boundary layer thickness $\delta$. Then, equating [11] and [16] and rearranging

329 gives

330

$$
k_{e q}=D_{V} \frac{\mu}{\rho}\left(\frac{C^{0}-C^{\infty}}{P_{C}^{0}-P_{C}^{\infty}}\right)
$$

331 where the variables with a superscript of 0 correspond to the cavity wall and those with a

332 superscript of $\infty$ denote the cavity air. The capillary pressure of the cavity $P_{C}{ }^{\infty}$ is equivalent to

333 the relative humidity of the cavity [3], as described by Kelvin's equation [2]. The vapor 334 concentrations are computed according to [9] and [10]. Equation [18] was implemented in

335 iTOUGH2 as an equivalent permeability for the special evaporation connections. Note that $k_{e q}$ is 
merely a numerical trick used to represent the concentration-gradient-based vapor diffusion equation using the capillary-pressure-gradient-based flow equations used in iTOUGH2. When the conditions for both evaporation and seepage permit, the total mass flow from the cavity wall to the cavity is considered as the sum of both.

\section{Numerical Meshes}

341 Different numerical models were constructed to simulate liquid-release tests and seepage into the 342 underground openings at different test locations. Three-dimensional meshes of the test sites were 343 generated with grid sizes of $0.3 \mathrm{~m} \times 0.1 \mathrm{~m} \times 0.1 \mathrm{~m}$ for the Cross Drift and $0.1 \mathrm{~m} \times 0.1 \mathrm{~m} \times 0.1 \mathrm{~m}$ 344 for Niche 5 (see Fig. 5). The total mesh size of the models was on the order of 100,000 345 gridblocks. For the Cross Drift meshes, a circular cylindrical cavity of $5 \mathrm{~m}$ diameter was

346 removed from the center of the mesh to represent the cavity. Only one half of the symmetric 347 mesh was used in the simulations to save computational load. For the Niche 5 meshes, surveyed 348 niche geometry was removed from the numerical mesh to replicate the test sites. The liquid349 release boreholes are indicated in Fig. 5 by bold black lines, and the white sections at the middle 350 of the boreholes represent the injection intervals. The Cross Drift borehole is inclined while the 351 Niche 5 boreholes are parallel to the centerline of the niche. The Cross Drift mesh in Fig. 5a 352 represents the Zone 2 test interval. In Fig. 5b and 5c, boreholes \#4 and \#5 are revealed, 353 respectively (see also Fig. $3 c$ ). Notice that the injection intervals in boreholes \#4 and \#5 are 354 located at 3-3.5 $\mathrm{m}$ and $8.5-8.8 \mathrm{~m}$, respectively, from the borehole collars; hence, the respective 355 niche outlines are different.

356 The spatial structure of the Niche 5 permeability data was analyzed using the GSLIB module 357 GAMV3 (Deutsch and Journel, 1992) and a spherical semivariogram was fitted to the resulting 358 variogram. 
In the Cross Drift six air-injection tests were performed in borehole intervals approximately 1.8

$360 \mathrm{~m}$ in length, providing an estimate of mean log-permeability at the experimental site of -10.73

361 (corresponding to a permeability of $1.86 \times 10^{-11} \mathrm{~m}^{2}$ ) with a standard deviation of 0.21 .

362 Variability in permeability on the scale of a gridblock (which is $0.3 \mathrm{~m}$ long) is expected to be

363 higher than the measured standard deviation. For the purpose of generating a heterogeneous

364 field, permeability is taken to be log-normally distributed with a standard deviation of one order

365 of magnitude. Because the number of data points was insufficient to reveal the spatial correlation

366 structure of the permeability field, a weak spatial correlation of $0.2 \mathrm{~m}$ was prescribed that is

367 consistent with the geostatistical results reported by Finsterle et al. (2003). The computed and

368 prescribed geostatistical parameters are reported in Table 1 . These parameters were used to

369 constrain generation of spatially correlated permeability fields, using the Gaussian sequential

370 indicator simulation (SISIM) module of the GSLIB (Deutsch and Journel, 1992). Multiple

371 realizations of the permeability field were generated and mapped to the numerical meshes.

372 Representative permeability field realizations for the Cross Drift and Niche 5 are shown in Fig.

3735.

374 The cavities were represented in the seepage models by two types of overlapping gridblocks, one

375 corresponding to seepage and the other to evaporation as shown in Fig. 4. The seepage

376 gridblocks were assigned a zero capillary pressure, whereas the evaporation gridblocks were

377 assigned a capillary pressure and vapor concentration corresponding to the cavity relative

378 humidity, as given by [2] and [3]. No-flow boundary conditions are specified at the left, right,

379 front, and back sides of the model. A free-drainage boundary condition is applied at the bottom

380 to prevent an unphysical capillary boundary effect. 


\section{RESULTS AND DISCUSSIONS}

\section{Evaporation Boundary Layer}

383 The evaporation data collected in Niche 5 were used to calibrate the evaporation model. The data

384 were grouped into three classes based on airflow velocity (ventilation): (1) inside Niche 5

385 without ventilation; (2) outside Niche 5 with active ventilation; and (3) outside Niche 5 without

386 active ventilation, the regime usually encountered during nights and weekends. In Fig. 6, the

387 measured relative humidity, and temperature, and evaporation rates from still water are plotted.

388 The evaporation model [12] was fitted to the measured data by adjusting the boundary layer

389 thickness. The relative humidity and temperature measurements were conducted at a different

390 time interval from the evaporation rate measurements. Thus, fitting was performed by matching

391 the calculated and measured evaporation rates averaged over selected short durations of

392 significance (such as continuous high or low evaporation rates). The estimates of the boundary

393 layer thickness rounded to the nearest millimeter are listed in Table 2.

394 In agreement with the theoretical assessment (Equation [7]), the estimated $\delta$ showed an inverse

395 relationship with the ventilation conditions. Inside Niche 5, the air was the calmest because it 396 was isolated from the Cross Drift by a bulkhead (see Fig. 3). As a result, the thickest boundary 397 layer $(20 \mathrm{~mm})$ was obtained inside Niche 5. Fig. 6 shows that the relative humidity outside Niche

3985 increases at nights and during weekends when active cavity ventilation is turned off. However, 399 this increase in relative humidity is insufficient to explain the observed decrease in evaporation. 400 Therefore, as shown in Table 2, reduced air ventilation during nights and weekends is also 401 accompanied by an increase in the thickness of the boundary layer. The estimated boundary402 layer-thickness values and Equation [7] suggest that the air velocity outside Niche 5 is higher 403 than the inside by factors of 7 (without active ventilation) and 16 (with active ventilation). 


\section{Coupled Seepage and Evaporation}

405 In this section, simulations of coupled seepage and evaporation are compared with measured 406 seepage rate data. The software iTOUGH2 (Finsterle, 1999) was used to match the simulated 407 seepage rate with the measured values by adjusting the free capillary strength parameter $(1 / \alpha)$ 408 (Finsterle et al., 2003). The corresponding evaporation rate from the cavity walls was simulated using the cavity relative humidity and calibrated boundary layer thickness.

\section{$410 \quad$ Niche 5}

411 Two different data sets from liquid release tests conducted in boreholes \#4 (October, 2002) and

412 \#5 (July 2002) are compared with the Niche 5 seepage models. The liquid release rate, seepage 413 rate, and relative humidity data as well as modeled liquid release rate and fitted seepage rate are 414 shown in Fig. 7. The best-fit $1 / \alpha$ values were $671 \pm 223 \mathrm{~Pa}$ and $740 \pm 339 \mathrm{~Pa}$ for boreholes $\# 4$ 415 (30 inversions) and \#5 (24 inversions), respectively. The measured seepage rates attained a steady-state flow rate after several days. Because the early-time transient data are biased by storage (e.g., in lithophysal cavities and matrix) and/or fast flow paths connecting the injection 418 interval to the cavity ceilings, the model was fitted to the late-time steady state data. In the 419 simulations, the relative humidity was kept constant at 0.85 to match with the lowest steady420 conditions observed during the borehole \#4 tests.

421 To quantify the impact of evaporation on seepage over the observed high relative humidity range

422 (0.85-0.99), the calibrated seepage model of borehole \#4 was used to simulate seepage and 423 evaporation at relative humidity values of $0.85,0.95$, and 0.99 . The resulting steady-state 424 seepage and evaporation rates (on Day 266) are plotted in Fig. 8. At a relative humidity of 0.85 , 425 the evaporation rate from the entire niche wall surface and the seepage rate are comparable in 426 magnitude. As the relative humidity was increased, the steady-state evaporation rate showed a 
drastic decrease, while the corresponding seepage rate increased only slightly. Note that on day

428266 storage of liquid in the porous medium does not play a role on the water balance because

429 steady-state flow is attained (see Fig. 7). Thus, these model results suggest that at high relative 430 humidity conditions the main impact of evaporation is on the quantity of liquid diverted around 431 the cavity.

\section{ECRB Cross Drift}

433 In this subsection, two different data sets from liquid release tests conducted in borehole LA2, 434 Zone 2, and Zone 3 are compared with the ECRB Cross Drift seepage model. The liquid release rate, seepage rate, and relative humidity data, as well as modeled liquid-release rates and fitted seepage rates, are plotted in Fig. 9. The best-fit capillary-strength parameters $(1 / \alpha)$ were $557 \pm$ $56 \mathrm{~Pa}$ for zone 2 and $535 \pm 58 \mathrm{~Pa}$ for zone 3, based on 21 and 19 inversions, respectively. Note

438 that both of the liquid-release tests were conducted concurrently. The measured and simulated seepage-rate fluctuations were strongly correlated to the drastic changes in relative humidity

440 (hence, evaporation). The model captured this evaporation effect satisfactorily, tracking 441 increases in measured seepage rates as relative humidity increased and vice versa.

442 The interplay between relative humidity fluctuation and dynamics of flow and ceiling wetness at 443 different times during the test in Zone 2 are visualized in Fig. 10. During this test, the liquid 444 release rate was relatively stable (steadily increasing from approximately $31 \mathrm{~mL} / \mathrm{min}$ on Day 296 445 to approximately $34 \mathrm{~mL} / \mathrm{min}$ on Day 327 ). However, the relative humidity fluctuated between $44630 \%$ and $90 \%$ during this period. Fig. 10 shows snapshots of the liquid saturation distribution on 447 Days 296, 306, 316, and 326. Just before the test began, the drift wall had dried out because of 448 the low relative humidity in the drift. The liquid saturation at this time was in equilibrium with 
449 the assumed background percolation flux of $2 \mathrm{~mm} / \mathrm{yr}$. On day 306 of injection (relative humidity $450-70 \%$ ), water reached the crown of the drift, seepage had started, water was being diverted 451 around the drift, and the wet plume had reached approximately midway around the drift. After 45220 days (day 316 ), however, the plume had shrunk significantly because of reduced humidity 453 (approximately 38\%) and increased evaporation. Moreover, the seepage rate and seepage 454 locations (indicated by inverted triangles) had decreased. On about day 326, the relative 455 humidity rose up to approximately $80 \%$; thus, the evaporation rate was reduced, the wet plume 456 grew, and seepage rate and number of seeps increased. In general, despite the high liquid release 457 rate, the flow regime remained unsaturated. The liquid saturation was highest near the drift 458 crown, which induces a capillary pressure gradient that promoted flow diversion around the drift 459 (capillary barrier effect). Seepage and evaporation removed water from the formation as water 460 flowed around the drift, limiting the spread of the wetted region on the drift wall.

\section{SUMMARY AND CONCLUSIONS}

462 In this paper, we (1) estimated the evaporative boundary-layer thickness by calibrating a semi463 physical evaporation model that considers isothermal vapor diffusion; (2) calibrated a 464 heterogeneous fracture-continuum model against seepage-rate data; and (3) tested the effect of evaporation on seepage predictions. The major conclusions of this study are listed below:

1. The simplified vapor-diffusion approach of modeling evaporation was found to be effective in capturing the roles of the important environmental conditions that affect evaporation conditions at the liquid-release test sites at Yucca Mountain. 
2. We found that evaporation reduces seepage significantly in tests conducted under ventilated

472 conditions. Therefore, it is important to account for evaporation effects when calibrating a seepage process model against liquid-release-test data collected under ventilated conditions. In contrast, the impact of evaporation on seepage rate was minimal in closed-off niches,

3. The classification of ventilation regimes is based on crude assessment of the cavity environment. The effect of external wind velocity variations (note that the Cross Drift is connected to the air outside the ESF) was not accounted for in the calculations. The match between measured evaporation rate and model predictions could have been improved if accurate measurement of air velocity in the cavities had been made during the test.

483 The calibrated seepage models were tested by comparing blind predictions of seepage rates 484 performed with the calibrated model against liquid release test data that were not used for model 485 calibration. These model tests showed that the models predict seepage rate successfully according to a probabilistic acceptance criterion, which requires that at least $95 \%$ of the observed

487 late-time seepage-rate data lie within the $95 \%$ simulation uncertainty band calculated by the 488 calibrated model, or if the calibrated model over-predicts the seepage rates (deemed conservative). The model testing exercises and acceptance criterion are discussed in depth by 490 Finsterle et al. (2003) and are not reported in this paper. 


\section{ACKNOWLEDGMENT}

492 Thorough reviews and insightful comments by J. Birkholzer, G. Li, C. K. Ho, R. Fedors, C 493 Oldenburg and an anonymous reviewer are gratefully acknowledged. This work was supported 494 by the Director, Office of Civilian Radioactive Waste Management, U.S. Department of Energy, 495 through Memorandum Purchase Order QA-B004220RB3X between Bechtel SAIC Company, 496 LLC, and the Ernest Orlando Lawrence Berkeley National Laboratory (Berkeley Lab). The 497 support is provided to Berkeley Lab through the U.S. Department of Energy Contract No. DE498 AC03-76SF00098. 